\title{
Réflexions sur la radiocancérogenèse
}

\author{
Raymond LATARJET *
}

(Manuscrit reçu le 19 septembre 1990)

\begin{abstract}
RÉSUMÉ A la lumière de faits biologiques dûment confirmés, touchant à la transformation cellulaire maligne et, plus particulièrement, à la radiocancérogenèse, on discute les bases sur lesquelles sont fondées les valeurs des doses de rayonnements ionisants actuellement considérées comme admissibles.

Cette mise en question entraîne de sérieuses conséquences sur des notions fondamentales, telle l'existence de seuils, sur les méthodes d'extrapolation des FF (fortes doses, forts débits) au ff (faibles doses, faibles débits), enfin sur la considération qu'on doit accorder aux fluctuations de la radiation naturelle.
\end{abstract}

ABSTRACT In the ligt of well-established biological facts concerning the malignant transformation of cells and, more particularly, radiation carcinogenesis, the bases on which the values of the doses of ionizing radiations presently permissible have been founded are discussed.

This casts doubts on the serious consequences of such foundamental questions as that of thresholds, the methods of extrapolation from HH (high doses, high dose-rates) to ss (small doses, small dose-rates), and finally on the consideration which must be granted to the fluctuations of natural radiation.

\section{INTRODUCTION}

Le rapport $n^{\circ} 23$ de l'Académie des sciences sur les "risques des rayonnements ionisants et normes de sécurité" [1] n'a été rédigé qu'après des discussions approfondies dont les détails n'ont évidemment pas été donnés. Ainsi, semble-t-il y avoir quelqu'intérêt à développer quelques-unes des considérations qui sous-tendent les conclusions de ce rapport.

Dans le présent article, je présente et argumente mes points de vue personnels dans les secteurs auxquels je me suis plus spécialement consacré. Parfois, mes positions apparaîtront assez abruptes à certains lecteurs. Je tiens à préciser qu'elles n'engagent que ma seule responsabilité.

* Institut Curie, 26, rue d'Ulm, 75005 Paris. 
A l'origine, les normes de sécurité furent fort justement fondées sur le soin de respecter l'équilibre génétique de l'espèce humaine, à travers les données quantitatives que l'on possédait sur la mutagenèse naturelle et radio-provoquée chez la souris et chez l'homme. La réunion internationale décisive se déroula à Stockholm en septembre 1952 [5]. Antoine LACASSAGNE et moi y représentions la France. La liste des participants ne manque pas d'impressionner aujourd'hui. On y trouvait en effet, entre autres : L. H. GRAY et M. W. MAYNEORD pour la Grande-Bretagne ; H.J. MULLER, M. L. RUSSELL et son épouse Liane, C. TOBIAS, Lauriston TAYLOR pour les USA ; R. M. SIEVERT, A. G. FORSBERG, B. LIONDELL pour la Scandinavie ; et puis B. RAJEWSKI (Allemagne), G. von HEVESY (Hongrie), Z, BACQ (Belgique). Deux personnalités dominèrent les débats, MULLER par sa "dimension" et RUSSELL pour l'ampleur et la rigueur de ses expériences sur la souris à Oak Ridge.

Les premières normes furent fondées essentiellement sur les données d'alors concernant :

- la radiomutagenèse chez la souris,

- la fréquence moyenne, évaluée à $8 \%$, des morts génétiques chez l'homme (pour environ 2000 maladies connues),

- l'extrapolation de la souris à l'homme,

- le postulat qu'en première approximation la radiomutagenèse peut être admise comme un phénomène stochastique $\left(^{*}\right)$.

Au cours des années suivantes, la Commission internationale de protection radiologique (CIPR) modifia ces premières normes en tenant compte des améliorations apportées aux valeurs admises pour ces données de base, et ce fut fort bien [4].

Plus récemment, la CIPR, I'UNSCEAR, le Committe on the biological effects of ionizing radiation (BEIR) se sont écartés de la voie raisonnable des mutations pour se porter sur la voie des cancers, laquelle se heurte à des difficultés et à des incertitudes beaucoup plus sérieuses, mais dont l'impact émotionnel sur le public est plus fort. Ce virage fut encouragé par le fait qu'Hiroshima-Nagasaki avaient apporté de précieuses données sur la radiocancérogenèse consécutive aux deux explosions, alors que la mutagenèse et la tératogenèse y étaient demeurées fort discrètes (ce qui, d'ailleurs, ne manque pas d'intérêt).

La nécessaire extrapolation des FF (fortes doses, forts débits) des explosions au ff (faibles doses, faibles débits) de la radioprotection doit alors couvrir l'intervalle maximal, c'est-à-dire comporter le maximum d'incertitudes dans la simplification à laquelle elle est condamnée. Cette extrapolation fut conduite selon un pseudo-raisonnement, véritable sophisme, que l'on trouvera explicité dans le paragraphe 44 du dernier projet de rapport de la CIPR, que l'on peut résumer de la façon suivante :

1. l'irradiation des cellules germinales fait muter ces cellules, la radiomutation germinale est un phénomène stochastique;

$\left({ }^{*}\right)$ Pour plus de détails, voir [11]. 
2. l'irradiation des cellules somatiques fait muter ces cellules, cette radiomutation somatique ne diffère de la précédente que par la nature de la cellule irradiée ; elle est donc également un phénomène stochastique ;

3. le cancer étant une mutation somatique, la radiocancérisation est donc stochastique ;

4. d'où une relation dose-effet simple, labsence de seuil, et des modèles d'extrapolation simples.

Malheureusement les propositions 2 et 3 sont erronées (j'ai souligné les erreurs). Précisons :

(2) Les deux catégories de mutations diffèrent bien par les cellules qui les subissent, ainsi qu'il est dit, elles diffèrent aussi par leurs mécanismes, et les différences sont telles que l'un étant stochastique n'entraîne nullement que l'autre le soit aussi (*).

(3) Un cancer n'est pas une mutation somatique ; il est un clone de cellules somatiques mutées.

En conséquence, on doit considérer les conclusions qui figurent en 4) comme non fondées. Pourtant, la CIPR les réaffirme et les propage depuis 25 ans comme des axiomes, au point que, aussi inexacts soientils, il est devenu aujourd'hui hérétique de les mettre en question.

Mon présent propos est précisément de les mettre en question à la lumière de faits expérimentaux dûment établis. Dans la première partie, je considérerai ceux qui se rapportent aux mécanismes de la radiocancérogenèse ; dans la seconde partie j'envisagerai briévement certains aspects des problèmes qui en dérivent concernant l'irradiation naturelle, le seuil et l'hormésis.

\section{QUELQUES FAITS DE RADIOCANCÉROGENÈSE}

Ne me faites pas dire ce que je ne dis pas. Bien entendu, la radiocancérogenèse commence par des interactions discontinues et aléatoires de l'énergie des rayonnements et de constituants cellulaires - l'"initiation " de BERENBLUM [2] - Ces interactions ont un caractère stochastique. Mais la cellule cancéreuse n'est pas encore née ; la tumeur cancéreuse encore moins. Cette seconde naissance n'est pas inéluctable ; la situation est réversible ; l'évolution vers la malignité est même encore très improbable; elle exige d'autres interventions. C'est là que le caractère stochastique va disparaître.

(*) Une balle dans la tête tue par effet à un coup. Un peu d'arsenic chaque soir dans la tisane tue également, mais par effet cumulatif. 
Examinons quelques situations en commençant par les plus simples (apparemment).

1. Plus nous avançons dans la connaissance, plus s'affirme l'évidence que les chemins de la radiocancérogenèse sont multiples et divers. Les séquences d'événements ne sont pas les mêmes qui conduisent à une tumeur solide, à une leucémie, à un mélanome, etc. II faut bien que ces chemins convergent quelque part puisqu'ils aboutissent au clone de cellules malignes ; mais où donc se situe ce point de convergence, et en quoi consiste-t-il ?

\section{Radiocancérisation in vitro}

Dans ces expériences, on irradie des cellules normales en culture, puis on les met en incubation. A chaque génération, on examine si de nouveaux caractères sont apparus, en particulier caractères morphologiques, perte de l'inhibition de contact, et surtout apparition de la malignité (par greffe de retour sur l'animal donneur des cellules initiales). Considérons, à titre d'exemple, les expériences bien connues de John LITTLE et coll. [12] sur les cellules 3T3 d'embryons de souris $\mathrm{C} 3 \mathrm{H}\left(^{*}\right)$ :

- les cellules de première génération (celles qui ont été irradiées) ne montrent aucune modification morphologique, elles ne sont pas cancéreuses;

- après quelques générations, la morphologie se modifie, tandis qu'apparaissent de nouveaux caractères de croissance ; l'inhibition de contact disparaît, mais ces cellules ne sont toujours pas cancéreuses (les greffes de retour sur l'animal donneur restant négatives) ;

- c'est seulement après d'autres générations, en nombre variable selon les conditions expérimentales, que la malignité apparaît et se "fixe";

- on ignore la nature biochimique des changements initiaux qui s'avèrent transmissibles et évolutifs.

Cette situation rappelle à maints égards celle des mutations différées, découvertes en 1946 par DEMEREC et LATARJET sur des bactéries irradiées [7].

Brièvement, on irradiait les bactéries avec des rayons $X$ ou des rayons UV. On déterminait le nombre de bactéries irradiées devenues résistantes à un bactériophage (mutations immédiates) ; puis, on mettait les bactéries en culture sur un milieu nutritif solide. A chaque génération, on voyait apparaître au sein des clones qui grossissaient (qu'on ne dérangeait pas) de nouveaux mutants (mutations différées dont on déterminait le nombre). Ce nombre augmentait jusqu'à la $13^{e}$ génération. Finalement, dans ces expériences, les mutations différées étaient 200 fois plus

(*) On lira avec intérêt dans [12] les conditions qu'on en peut tirer vis-à-vis de la cancérisation in vivo. 
nombreuses que les mutations immédiates. Le mécanisme de ce phénomène, dûment confirmé depuis, n'a jamais été élucidé. II présente d'évidentes analogies avec la radiocancérogenèse in vitro, et offre un système expérimental plus simple.

\section{Les réparations fautives}

Pendant une quarantaine d'années, la cancérogenèse expérimentale s'est heurtée à une question fondamentale qui l'embarrassait fort : la condition première pour une cellule transformée de conduire à une tumeur est de proliférer, c'est-à-dire d'être viable. Or, les rayonnements et les produits chimiques cancérogènes sont cytocides; ils tuent les cellules. Comment produire des tumeurs si on commence par tuer les cellules ? La découverte des réparations fautives au cours des années 60 a donné une première réponse satisfaisante à cette question.

Rappelons quelques données quantitatives sur la cinétique de la synthèse de l'ADN dans les cellules humaines. Les dix milliards de nucléotides qui constituent cette molécule sont alignés, selon un ordre rigoureux, en une fibre superenroulée qui, une fois déroulée, a une longueur d'environ $2 \mathrm{~m}$ pour un diamètre moyen de 2,2 $\mathrm{nm}$. Au cours du cycle de division cellulaire, la période dite $S$ au cours de laquelle la nouvelle molécule d'ADN est synthétisée, dure au plus 6 heures. Ainsi, 400000 nucléotides sont synthétisés par seconde, la molécule mère se déroulant au rythme de 50 tours par seconde $\left(^{*}\right)$.

Si on considère la complexité chimique du nucléotide, et la nécessité de le synthétiser à partir des éléments relativement simples fournis par la digestion des aliments, on saisit l'extraordinaire chimie qui se deroule en nous à une vitesse sans commune mesure avec celles de notre chimie industrielle, et pour cette seule synthèse de l'ADN. C'est un des aspects du miracle de la vie.

On conçoit également qu'au cours de ces processus si rapides et si délicats quí se déroulent au sein d'un protoplasme agité, des erreurs solent inévitables, et comme la continuité exige que la copie reste parfaite, il est indispensable que la cellule soit dotée de puissants mécanismes qui reconnaissent et réparent les erreurs. Tel est bien le cas. La cellule possède des enzymes de réparation, dites constitutives, qui jouent ce rôle à la perfection, en ce sens qu'elles effacent parfaitement les erreurs, assurant la restitutio ad integrum.

Mais il peut arriver - et c'est le cas lorsque la cellule est attaquée de l'extérieur, notamment par un agent cytocide - que les erreurs provoquées soient trop nombreuses pour que les enzymes constitutives suffisent à la tâche. Retentit alors dans la cellule une alarme, un véritable SOS, auquel elle répond par la formation de nouvelles enzymes de réparation, dites inductibles. Celles-ci, à leur tour, réparent des erreurs, mais

${ }^{*}$ ) Au total, chez l'homme de $70 \mathrm{~kg}$, la longueur totale de l'ADN synthétisé chaque jour est de l'ordre de 200 millions de kilomètres, soit du même ordre que la distance moyenne de la terre au soleil, et ceci ả partir de la ration alimentaire quotidienne. 
souvent de façon imparfaite. Certes, elles rétablissent la viabilité, mais en laissant des fautes non létales transmissibles. La réparation SOS est fautive ; elle est mutagène. On concoit maintenant que de telles fautes transmissibles puissent être à l'origine de processus conduisant à la malignité.

Telle est la réponse donnée à la question posée au début de ce paragraphe. Cette réponse (sur laquelle on reviendra plus loin à propos de I'hormésis) satisfait d'autant mieux que les rayonnements sont un excellent inducteur des réparation fautives $\left(^{*}\right)$.

\section{Les effets de promotion}

On vient de voir qu'entre les modifications moléculaires initiales et l'apparition du caractère malin, une plus ou moins longue période s'écoule - qui peut être très longue, plusieurs décennies parfois chez l'homme - couvrant de nombreuses générations cellulaires, lesquelles subissent une cascade de modifications progressives. C'est au cours de cette période, dite de latence, alors que la situation est encore réversible, que des influences diverses peuvent s'exercer qui décideront de l'issue de l'évolution amorcée.

Pour simplifier, je distinguerai trois types d'influences.

a) Les promoteurs. II existe des substances ou des circonstances pathologiques, non cancérogènes par elles-mêmes, qui favorisent l'expression de l'effet cancérogène d'un agent reconnu comme tel, en orientant vers la malignité le processus amorcé, ou en l'accélérant. On doit à BERENBLUM et SHUBIK [2] d'avoir clairement distingué le rôle "initiateur" du cancérogène, qui déclenche le processus, de ce rôle de "promoteur" du non cancérogène (dans leurs premières expériences, le promoteur était l'huile de croton dont le principe promoteur fut ensuite identifié comme étant l'acétate de phorbol $^{\star *}$ ).

Pour la radiocancérogenèse, la situation est particulièrement complexe du fait que les rayonnements sont à la fois initiateurs et promoteurs. Aussi, n'est-il pas étonnant que la plupart des radiocancers (pas tous, bien sûr, voir Hiroshima-Nagasaki) résultent d'irradiations répétées, par un long processus au sein duquel il n'est guère facile de distinguer ce qui ressortit à initiation et à promotion. Ceci n'empêche pas l'intervention possible d'autres promoteurs (chimiques) après une ou plusieurs irradiations initiatrices.

(*) Les principaux travaux initiaux sur les réparations fautives dont on trouvera une revue dans [21], sont dus à Evelyn WITKIN, à Miroslav RADMAN [15], à Raymond DÉVORET, et, plus récemment à Robert FUCHS. A leur origine est la découverte de I" UV-réactivation" par le Suisse Jean WEIGLE [20] qui travaillait alors à Pasadena.

(**) En fait, du point de vue historique, ce sont LACASSAGNE et VINZENT qui, les premiers, mirent en évidence un effet de promotion, celui de l'inflammation chez le lapin irradié localement par les rayons $X[10]$. 
Ces promotions nous amènent à évoquer les possibilités de potentiations souvent très puissantes, comme, par exemple, la potentiation entre amiante et tabac dans la production des cancers du poumon pour lesquels on a observé les fréquences relatives suivantes :

$\begin{array}{ll}\text { tabac seul } & : 10 ; \\ \text { amiante seule } & : 1 ; \\ \text { tabac + amiante } & : 70 .\end{array}$

II est certain que la diversité des réactions individuelles après une irradiation de masse (Hiroshima-Nagasaki) - même entre individus exposés aux mêmes doses dans des conditions comparables - s'explique, en grande partie, par la diversité des effets de promotion ou de potentiation que ces individus ont ultérieurement subis.

b) Les facteurs endogènes de risque. Les promoteurs et potentiateurs que l'on vient de voir constituent en quelque sorte des "facteurs exogènes de risque". En regard, existent des facteurs endogènes de risque. Nous en avons vu plus haut un bel exemple avec les enzymes de réparation des radiolésions de l'ADN. Les individus génétiquement appauvris en ces enzymes sont plus exposés que les autres à l'action cancérogène des radiations. Par exemple (il y en a d'autres), les sujets atteints de xeroderma pigmentosum, redoutable cancer de la peau produit par le rayonnement ultraviolet solaire, sont héréditairement déficients en enzymes de monomérisation des dimères de pyrimidines, lésion initiatrice produite par les rayons UV dans I'ADN (3).

II existe, certainement, bien d'autres facteurs endogènes de risque, un domaine sur lequel on vient seulement de se pencher. Voici, à titre d'exemple, un cas clair de cancérogenèse chimique. On s'est demandé depuis longtemps pourquoi certains grands fumeurs ne développent pas de cancer du poumon. On a récemment trouvé, dans les homogénats de poumons humains, un cytochrome inductible, appelé $P 450 \mathrm{IA}$, plus ou moins inductible selon les individus. Ce cytochrome métabolise certains cancérogènes de la fumée du tabac, les amines aromatiques et les hydrocarbures polycycliques, en produits éliminés par l'urine. Les individus les plus riches en ce cytochrome sont ainsi plus résistants à la cancérisation du poumon par le tabac.

c) Actions synergiques. Une autre circonstance illustre bien la complexité de la cancérogenèse. Il arrive parfois qu'elle demande les interventions conjointes - on peut dire synergiques - de plusieurs agents si différents qu'aucune relation n'apparaît entre eux. Tel est le cas du cancer du nasopharynx d'Extrême-Orient. Des études épidémiologiques récentes [19] ont montré que sa production exige la co-opération de trois facteurs : un virus à ADN du type Epstein-Barr, un génotype particulier (encore non précisé) du sujet en cause ; enfin la coutume, pour ce sujet, de manger fréquemment des poissons non vidés ! On ne voit pas bien comment pareille situation s'accomoderait d'un schéma simpliste construit autour d'un postulat de stochastisme. 


\section{Le combat contre la croissance tumorale}

La cellule cancéreuse n'est pas un cancer. Un cancer, je l'ai déjà dit, est une colonie (un clone) issue d'une ou de plusieurs cellules malignes. Pour qu'un cancer s'installe en situation de non-retour, et devienne observable, il faut que la colonie se développe au-delà d'une certaine dimension ; ce développement se heurte à de nombreux obstacles que l'organisme lui oppose, fort heureusement d'ailleurs, sans quoi nous serions tous cancéreux, un jour ou l'autre.

La mieux connue aujourd'hui des réactions que l'organisme oppose à la croissance tumorale est sans doute de type immunologique (il y en a d'autres). Le caractère cancéreux se manifestant notamment par la présence de nouvelles protéines dans la cellule, cette cellule est antigénique, c'est-à-dire capable de susciter diverses réactions d'immunité de la part de l'hôte - d'où dérivent aujourd'hui divers modes d'immunothérapie de certains cancers. La place n'est pas ici de s'étendre sur ce nouveau domaine. Les termes de lymphocytes $T$, de cellules tueuses, d'interféron, de lymphokines (la fameuse interleurkine II en particulier) sont déjà bien connus.

\section{Les oncogènes}

On ne saurait terminer cette rapide revue sans parler des oncogènes, champ qui, par récente explosion, est devenu sans doute le plus dynamique de la recherche cancérologique actuelle.

Depuis une trentaine d'années, on sait que les virus cancérogènes doivent, en partie, leur action à ce que leur génome contient des gènes, dits oncogènes, qui, une fois intégrés dans le génome de la cellule infectée, codent pour de nouvelles protéines. Celles-ci, les kinases, de faible poids moléculaire, confèrent à cette cellule de nouveaux caractères au niveau des fonctions qui régissent la régulation de la croissance cellulaire, c'est-à-dire la perméabilité de la membrane, le transport de l'information de cette membrane au noyau, etc.

Vers 1975, il a été démontré que ces gènes sont d'origine cellulaire. Ils existent dans la cellule sous une forme inactive ou strictement régulée, les proto-oncogènes que les virus ont "empruntés" [17]. On connaît aujourd'hui une centaine de proto-oncogènes. Ceux-ci peuvent être "activés" en oncogènes par divers types d'interventions, notamment par des rétrovirus. Cette activation peut simplement résulter d'une mutation par modification de la séquence nucléotidique sur des codons spécifiques, d'où le codage de nouvelles protéines. Elle peut avoir des origines plus complexes. La transformation maligne de la cellule exige l'intervention de plusieurs oncogènes.

II existe, en outre, des anti-oncogènes, gènes qui s'opposent à l'action des oncogènes et empêchent ainsi l'expression des caractères de malignité provoquée par ceux-ci. L'apparition d'un cancer peut ainsi suivre soit l'activation d'un proto-oncogène, soit l'inhibition d'un anti-oncogène, lorsque l'effet de celui-ci est dominant. C'est le cas du rétinoblastome, redoutable cancer de l'œil qui survient le plus souvent chez de jeunes enfants. 
Les altérations chromosomiques, en particulier les remaniements au niveau de proto-oncogènes et les délétions au niveau des antioncogènes, constituent des événements rares propres à entraîner la cancérisation. D'où un mécanisme concevable pour certains cancers produits par les rayonnements, encore que rien ne soit clair pour l'instant à ce sujet.

D'ailleurs, plus généralement, et bien que leur mode d'action soit encore fort obscur, les oncogènes offrent un possible point de convergence (tant attendu !) aux si divers modes de cancérisation par virus, par radiations, et par composés chimiques.

Cette brève excursion parmi quelques faits de radiocancérogenèse permet de dégager les points suivants :

- l'extrême complexité des processus de cancérisation, en particulier par les rayonnements ;

- la diversité des chemins possibles qui conduisent de l'absorption de l'énergie de radiation par l'organisme à l'émergence d'un cancer ;

- la multiplicité des éventuelles interventions, non cancérigènes par elles mêmes, qui, au long de ces chemins, décident si le processus engagé aboutira ou non à un cancer ;

- en conséquence, réduire en postulat l'ensemble de ces processus à des concepts et modèles ultra-simplifiés est une attitude dangereuse contraire à l'objectivité scientifique, qui ne peut conduire qu'à des conclusions a priori inexactes. C'est pourquoi notre rapport n'a pas caché ses réserves vis-à-vis de recommandations fondées sur de tels concepts et modèles ultra-simplifiés.

Je précise plus brutalement qu'à mon avis personnel, contrairement au dogme de la CIPR, et pour tous les motifs qui viennent d'être exposés, la radiocancérisation n'est pas (au moins dans la plupart des cas) un phénomène stochastique, et que les conclusions tirées a priori de ce dogme ne peuvent pas être acceptées.

\section{QUELQUES POINTS SENSIBLES : SEUIL, IRRADIATION NATURELLE, HORMESIS}

Cet article a pour but, rappelons-le, de préciser ou de développer certaines considérations qui sous-tendent les positions prises par l'Académie dans son rapport sur les normes de radioprotection. Les trois sujets mentionnés dans le titre ci-dessus méritent quelques remarques particulières étant donné l'importance qui leur a été dévolue dans le rapport. 


\section{Le problème de seuil}

Existe-t-il un seuil en radiocancérogenèse ? Question importante. S'il en est un, en-deçà de la dose seuil, le risque est nul. S'il n'en est pas, le risque n'est jamais nul, aussi faible soit la dose.

A la question posée, la CIPR répond par la négative. "II n'y a pas de seuil", affirme-t-elle en dogme. C'est la conséquence directe du dogme $n^{\circ} 1$ selon lequel la radiocancérogenèse est un phénomène stochastique $\left(^{*}\right)$. Ce dogme étant fondé sur des considérations inexactes, la question du seuil reste posée. Transférons la du dogmatisme à l'expérimentation.

Celle-ci nous offre maints exemples dans lequels se manifestent à l'évidence non seulement des seuils mais des seuils très élevés. Considérons l'un de ces exemples, la radioleucémie lymphoïde de la souris, non seulement parce que, parmi les cancers radio-provoqués, les leucémies tiennent une place importante, non seulement parce que le processus, très complexe, a été déchiffré dans ses détails, mais surtout parce qu'il démontre combien fallacieux sont les schémas simplistes actuellement acceptés.

De nombreux travaux, en particulier ceux d'Egon LORENTZ, ceux de Henri KAPLAN, ceux de Jean-François DUPLAN, ont démontré que la séquence suivante se déroule pour aboutir à près de $100 \%$ de leucémies chez certaines souches de souris (C3H, C57 BI...) lorsqu'on les soumet en totalité à des rayons $X$ à doses convenables (pour une revue d'ensemble, voir [9]). Cette séquence comporte quatre phases :

a) Les rayons $X$ détruisent les cellules de tissus lymphoïdes, du thymus notamment, qui sont les plus radiosensibles. La dose de radiation doit être suffisante pour que le dépeuplement lymphoïde soit quasi-total (sinon, ou si, après une dose suffisante, on injecte à l'animal des cellules lymphoïdes non irradiées, le processus leucémogène s'arrête là). D'où la nécessité d'un seuil élevé. Ce dépeuplement cellulaire, par quoi le processus commence, est un phénomène évidemment non stochastique. C'est ce que la CIPR se propose désormais d'appeler un effet déterministe (voir remarque à la fin de ce paragraphe).

b) La destruction des cellules thymiques déclenche la synthèse de facteurs humoraux ou hormonaux qui stimulent la régénération du thymus.

c) Sous son effet, le thymus commence à se reconstituer. Les nouvelles cellules thymiques, contrairement aux cellules habituelles,

(*) La CIPR reconnaît un cas particulier où un seuil peut être admis (sans pour autant contrevenir au dogme $n^{\circ} 1$ ). Il est bien établi que la latence d'apparition des cancers aug* mente lorsque la dose de radiation inductrice diminue. II arrive que la dose soit suffisamment faible pour que cette latence excède la longévité de l'irradié. Le radiocancer n'a plus le temps d'apparaître. Dès lors, cette dose est un seull. C'est évident. Mais ce cas particulier ne change rien au problème général du seuil qu'il tend à escamoter. En revanche, l'absence de seuil est théoriquement concevable dans le cas d'un sujet chez qui les conditions promotrices non aléatoires et nécessaires (présence d'un virus, activation d'un oncogène, etc., etc.) seraient remplies avant l'événement initiateur aléatoire. 
s'avèrent "compétentes" pour un virus, hôte normal de ces souris (virus à ARN du type virus de Gross), qui, jusque là, se tenait tranquille, mais qui trouve dans ces cellules de néo-formation un terrain propice à sa multiplication.

d) Le virus se multiplie, diffuse, envahit la totalité du tissu lymphoïde (rate, thymus, ganglions) et le cancérise ; d'où leucémie lymphoïde.

En ce qui concerne les doses de radiation, il faut d'abord franchir un seuil élevé, condition première puisque, comme nous venons de voir, le processus ne se déclenche que si l'aplasie lymphoide est quasi totale. Ainsi, une dose unique de 1 à 2 Gy (100 à 200 rads) ne fait rien. Une dose unique de 7 Gy (700 rads) tue la plupart des animaux. Mais si on administre cette dose en 4 fractions de 1,75 Gy (175 rads) à une semaine d'intervalle, on ne tue pas, et $80 \%$ des souris développent la leucémie.

L'exemple de ce radiocancer nous conduit aux trois observations suivantes qui le concernent :

- le processus n'est pas stochastique,

- il comporte un seuil, un seuil très élevé,

- la courbe dose-effet, que l'on n'a pas déterminée (si tant est qu'on puisse le faire) n'aurait rien de commun avec les modèles simplistes que l'on se plait à discuter : linéaire, quadratrique, linéaire-quadratique, etc.

Les exemples ne manquent pas d'autres radiocancers très importants auxquels les trois mêmes conclusions s'appliquent. Citons les épithéliomas et sarcomes sur radiodermites chroniques et radionécroses qui ont alimenté le martyrologe des premiers radiologistes pendant une quarantaine d'années (1900-1940). Citons également les cancers cutanés produits par les rayons ultraviolets (lesquels, soit dit en passant, furent les premiers radiocancers décrits) $\left(^{*}\right)$.

Après 50 ans de recherches personnelles et de documentation de par le monde sur la radiocancérisation, j'aboutis aux conclusions suivantes concernant le problème de seuil :

- J'ignore s'il y a des radiocancers sans seuil ; je suis prêt à l'admettre si l'on m'en donne la démonstration.

- Je sais qu'il y a des radiocancers à seuil. Le seuil n'est pas le même pour tous ces cancers. II est parfois très élevé. Pour ceux-là, une fois le seuil franchi, la fréquence des cancers augmente très vite avec la dose, et la courbe dose-effet si on pouvait la déterminer, ne répondrait à aucune formule simple, la sensibilité individuelle à un cancer radio-induit variant au sein d'une population apparemment homogène.

(*) La même situation, à savoir seuil élevé et courbe dose-effet ne répondant à aucune relation mathématique simple, a été observée pour d'autres effets que la cancérogenèse. Ainsi, chez la souris irradiée in utero une dizaine de jours après la conception, la dose de $1 \mathrm{~Gy}$ (100 rads) ne produit aucun effet, ni décès, ni malformation osseuse. A 2 G (200 rads), $75 \%$ des animaux meurent après la naissance sans présenter de malformation. A 3 Gy (300 rads), tous les animaux meurent, dont $70 \%$ portent des malformations [13]. 
- Chez l'homme, la situation est obscurcie par un bruit de fond très intense (25-28\%) dans la responsabilité duquel l'alimentation, la pollution chimique génotoxique, et les réparations fautives naturelles de l'ADN jouent un grand rôle.

- Finalement, envisager le problème du seuil d'un point de vue théorique est un faux problème, une voie sans issue (le sexe des anges ?). Ce qui importe, c'est le point de vue pratique. On facilitera beaucoup le choix des normes de doses admissibles raisonnables en renonçant à discuter sans fin du problème théorique du seuil pour se rallier au concept de "seuil pratique", dose au-dessous de laquelle la probabilité de radiocancérisation devient négligeable en regard des autres causes de cancérisation.

A cet égard, l'omniprésence de la radiation naturelle doit faciliter les choses. Considérons-en donc maintenant quelques aspects.

Remarque. Dans le paragraphe $\mathbf{2 0}$ de son projet de recommandations 1990, la CIPR prend la décision non innoncente de remplacer l'expression "non stochastique" par "déterministe", et donne pour exemple d'effet déterministe la destruction des cellules d'un tissu. Le résultat de cette décision, sans doute son but, est un nouveau sophisme, à savoir que tout ce qui n'est pas déterministe devient stochastique $\left(^{*}\right)$. Rappelons à la CIPR que le contraire de "dêterministe" n'est pas "stochastique", mais "probabiliste".

\section{La radiation naturelle}

Le rapport de l'Académie des sciences a ménagé une large place à l'irradiation naturelle, notamment au problème du radon dans les habitations. Inutile d'y revenir. Je me borne à souligner ici deux points qui trouvent leur place aux côtés du seuil et de l'hormésis au sein de ce chapitre.

a) Que représente, en quantité, cette radiation naturelle ? Prenez la valeur moyenne annuelle de $2,4 \mathrm{mSv}$ (240 mrem) admise par l'Académie, et faites le calcul. Mon calcul m'a conduit à ce que, chez un homme de $70 \mathrm{~kg}$, cette dose équivaut à environ un milliard d'ionisations par seconde. C'est peu et c'est beaucoup. C'est peu si l'on considère le nombre colossal de molécules candidates à ces ionisations ; c'est beaucoup si l'on est de ceux que quelques ionisations effraient (**).

En tout cas, cette radiation constitue bien un élément permanent non négligeable de notre environnement naturel. L'évolution a dû en être influencée. Nous y reviendrons plus loin.

b) Le rapport de l'Académie a justement insisté sur les variations géographiques de lirradiation naturelle, tant avec l'altitude (rayons cosmiques) qu'avec la nature des sols (rayons telluriques). Dans les régions

(*) Je décide de remplacer "non chaud" par "tiède". En conséquence, ce qui n'est pas tiède devient chaud ( $y$ compris ce qui est froid).

(**) En 60 ans, compte tenu de l'évolution de son poids pendant les 20 premières années, notre sujet aura été le siège d'environ $1,5.10^{18}$ ionisations. 
dont les habitations sont construites en granite, les émanations de radon amplifient ce second facteur de variation. Si l'on excepte quelques points chauds d'étendue relativement faible sur des terrains fortement radioactifs, les variations de l'irradiation naturelle s'étendent sur une gamme de 1 à $3(1,50$ à $4,50 \mathrm{mSv}, 150$ à 450 mrem par an).

Il s'imposait de rechercher si ces variations se répercutent sur les hémopathies malignes, sur les maladies génétiques, et sur les cancers au sein des populations de ces régions. De nombreuses enquêtes épidémiologiques $\left({ }^{*}\right)$ effectuées de par le monde pour répondre à cette question, il ressort qu'à travers la disparité des réponses se dégage une moyenne, un centre de gravité très nets : non, il n'y a pas de répercussion. En d'autres termes, la zone des fluctuations de l'irradiation naturelle se trouve au-dessous de ce qu'on devrait considérer comme un seuil pratique $\left(^{* \star}\right)$.

\section{Hormésis}

On sait depuis toujours que des substances bienfaisantes deviennent dangereuses si on les absorbe à trop fortes doses (il ne faut abuser de rien). Claude BERNARD a popularisé cette notion dans son expression inversée, à savoir que de nombreuses substances, toxiques à fortes doses, se révèlent bénéfiques à faibles doses. La pharmacopée abonde en toxiques. En serait-il de même pour les radiations ionisantes ? ${ }^{* * *}$ )

La CIPR a répondu jusqu'ici par la négative, selon le dogme de l'absence de seuil, lequel stipule qu'une dose aussi faible soit-elle, comporte un risque non nul qui décroît avec la dose. Nous venons de voir dans les deux paragraphes précédents que cette thèse n'est plus admissible, et qu'au dessous d'un certain "seuil pratique", les radiations ionisantes doivent être considérées comme dépourvues de risque. Mais peut-on aller plus loin et penser que les faibles doses pourraient être bienfaisantes, idée couverte par le terme d'hormésis ?

Dans son dernier projet de rapport (1990), la CIPR a commencé d'assouplir sa position par un discret paragraphe $\left(n^{\circ} 45\right)$. Elle reconnaît que les radiations "peuvent stimuler certaines fonctions cellulaires telles que la réparation des radiolésions, certaines réponses immunologiques et hormonales, et raffermir ainsi les mécanismes naturels de défense de l'organisme". Elle ajoute, toutefois, que "ces effets restent mal compris et discutables, que la plupart des résultats expérimentaux ne permettent pas de conclure, et qu'en conséquence il n'est pas actuellement adéquat de penser que l'hormésis puisse influencer la probabilité d'induction d'effets stochastiques par les radiations".

$\left(^{\star}\right)$ Ces travaux sont trop nombreux pour être cités ici. Le lecteur pourra consulter les références $17,18,19$.

$\left(^{* *}\right)$ C'est d'alleurs ce que fait le bon sens populaire sans l'avouer. Tandis que certains s'émeuvent à l'idée que la centrale nucléaire de Fessenheim pourrait envoyer quelques dizaines de millirems par an sur la plaine d'Alsace, personne ne propose d'évacuer les proches vallées vosgiennes qui en reçoivent naturellement beaucoup plus.

(***) Pour les rayonnements ultraviolets, la réponse est oui : cancérogènes à fortes doses, anti-rachitiques à doses modérées, etc., etc. 
Dans son rapport, L'Académie des sciences, sans toutefois conclure, a retenu quatre raisons de prendre le problème de l'hormésis au sérieux.

a) La vie s'est développée dans le champ ininterrompu des rayonnements ionisants naturels, cosmiques, telluriques et organiques. Actuellement, la valeur moyenne de la dose annuelle reçue au niveau de la mer est de 2,4 milligrays $\left(^{*}\right)$. Comme on a vu au paragraphe précédent, elle correspond à environ un milliard d'ionisations par seconde chez un homme de $70 \mathrm{~kg}$. Ce n'est pas négligeable. Il est raisonnable de penser que cette irradiation permanente a joué un rôle dans la sélection naturelle, et que les espèces et races qui en ont émergé lui sont bien adaptées.

b) On a vu précédemment $\left(\mathrm{II}, 3^{\circ}\right)$, et la CIPR le reconnaît, que les rayonnements sont un excellent inducteur de la réparation SOS, c'està-dire qu'ils induisent un moyen de réparer les lésions produites dans I'ADN par elles-mêmes et, sans doute, par des agents chimiques génotoxiques du type dit "radiomimétique".

c) Le sens qu'on peut donner à l'expression d'effets bénéfiques n'est pas évident. II n'est pas nécessairement le même si l'on s'adresse à un individu ou à une population. Dans ce second cas, qui est le plus important, je serais enclin à qualifier de bénéfique tout effet quil confère un avantage sélectif à cette population. II convient alors de prendre en considération les travaux poursuivis à Toulouse depuis une quinzaine d'années par Hubert PLANEL et son équipe [14]. Selon leurs résultats, d'abord contestés mais aujourd'hui admis, les faibles doses administrées à faible doses administrées à faible débit par l'irradiation naturelle, augmentent le taux de croissance de populations de micro-organismes monocellulaires (paramécies notamment).

d) Plus proches de nous, plus "parlants", donc plus suggestifs, sont les résultats d'études statistiques où l'on a comparé les fréquences des cancers dans deux régions différant autant que possible par le niveau de l'irradiation ambiante, et aussi semblables que possible quant aux autres facteurs susceptibles d'influencer la cancérisation (alimentation, tabagisme, pollutions génotoxiques, (...) etc.). Par exemple, on a mis côte à côte une zone granitique et une zone alluviale, où compte tenu de la concentration en radon dans les habitations, l'irradiation ambiante différait de plus de deux fois (8). On a également mis côte à côte des zones différant par leur distance à une centrale nucléaire soupçonnée d'être responsable de détriments au sein de la population alentour (6).

Dans la plupart de ces études, on n'a pas observé d'augmentation de la fréquence des cancers dans les zones les plus irradiées. Bien plus, dans quelques cas, le résultat fut contraire à celui que la plupart attendaient, à savoir significativement moins de cancers dans ces zones.

(") Ici en grays et non en sieverts car ces derniers sont dérivés des grays, eux-mêmes dérivés de mesure d'ionisations. Il est d'ailleurs plaisant de noter que grays et sleverts, qu'on utilise, représentent des grandeurs que l'on ne mesure pas, faute de pouvoir le faire. Ce qu'on mesure, ce sont des ionisations, c'est-à-dire des roentgens, que l'on n'utilise plus, d'où I'on dérive les deux autres grandeurs par le calcul. 
Certains voient là l'effet de facteurs contingents non contrôlés, mais l'épidémiologiste honnête ne prend pas position a priori.

Remarque. Si les effets d'hormésis devaient être prouvés, le dogme de l'absence de seuil perdrait toute signification.

\section{BIBLIOGRAPHIE}

[1] ACADEMIE DES SCIENCES - Risques des rayonnements ionisants et normes de radioprotection. Paris : Académie des sciences, 1989 (Rapport no 23).

[2] BERENBLUM I., SHUBIK P. - A new quantitative approach to the study of the stages of chemical carcinogenesis of the mouse's skin. Brit. J. Cancer, 1947, 1, 383-391.

[3] CLEAVER J.E. - Defective repair replication of DNA in xeroderma pigmentosum. Nature, 1968, 218, 652-656.

[4] COMMISSION INTERNATIONALE DE PROTECTION RADIOLOGIQUE (CIPR) Recommendations. Oxford : Pergamon press, 1966 (ICPR publication 9).

[5] Conference on radiobiology and radiation protection. Acta Radiol., 1954, 41, $1-108$.

[6] COOK-MOZAFFARI P.J., DARBY S.C., DOLL R., FORMAN D., HERMON C., PIKE M.C., VINCENT T. - Geographical variation in mortality from leukemia and other cancers in England and Wales in relation to proximity to nuclear installations 1969-1978. Brit. J. Cancer, 1989, 59, 476-485.

[7] DEMEREC M., LATARJET R. - Mutations in bacteria induced by radiations. Cold Spring Harbor Symp. Quant. Biol., 1946, 11, 38-50.

[8] DOUSSET M. et JAMMET H. - Comparaison de la mortalité par cancer dans le Limousin et le Poitou-Charentes. Radioprotection, 1985, 20,61-67.

[9] KAPLAN H.S. - Leukemia and lymphoma in experimental and domestic animals. Ser. Haematol., 1974, 7, 93-163.

[10] LACASSAGNE A. et VINZENT R. - Action des rayons $X$ sur un foyer infectieuX local provoqué chez le lapin. C.R. Soc. Biol., 1929, 100, 247-248.

[11] LATARJET R. - Regulation of radiation pollution : its possible usefulness in strategy for intervention against chemical mutagens. IARC Scientific Publications, $1979,25,207-233$.

[12] LITTLE J.B. - The relevance of cell transformation to carcinogenesis in vivo. In : "Low dose radiation : biological basis of risk assessment". Londres : Taylor and Francis, 1989, pp. 396-413.

[13] MOLE R.H. - Irradiation of the embryo and fetus. Brit. J. Radiol., 1987, 60, 17-31.

[14] PLANEL H., SOLEILHAVOUP J.P., TIXADOR R., RICHOILLEY G., CANTER A., CROUTE F., CARATERO C., GAÜBIN Y. - Influence on cell proliferation of background radiation or exposure to very low chronic gamma radiation. Health Phys., 1987, 52, 571-578. 
[15] RADMAN M. - is there SOS induction in mammalian cells ? Photochem. Photobiol., $1980,32,823-830$.

[16] SOCIÉTÉ FRANCAISE DE RADIOPROTECTION (SFRP) - Exposition au radon dans les habitations : aspects techniques et sanitaires, Paris, 11-14 janvier 1989. Radioprotection, 1989, 24, 287-366.

[17] STEHELIN D., WARMUS H.E., BISHOP J.M., VOGT P. - DNA related to the transforming gene(s) of avian sarcoma viruses is present in normal avian DNA. Nature, 1976, 34, 2835-2841.

[18] TERZAGHI M., LITTLE J.B. - X-radiation induced transformation in a $\mathrm{C} 3 \mathrm{H}$ embryo derived cell line. Cancer Res., 1976, 36, 1367-1374.

[19] THÉ Guy de - Sur la piste du cancer. Paris : Flammarion, 1984.

[20] WEIGLE J. - Induction of mutations in a bacterial virus. Proc. Natl. Acad. Sci. USA, 1953, 39, 628-636.

[21] WITKIN E.M. - Ultraviolet mutagenesis and inducible DNA repair in Escherichia coli. Bacteriol. Rev., 1976, 40, 869-907. 